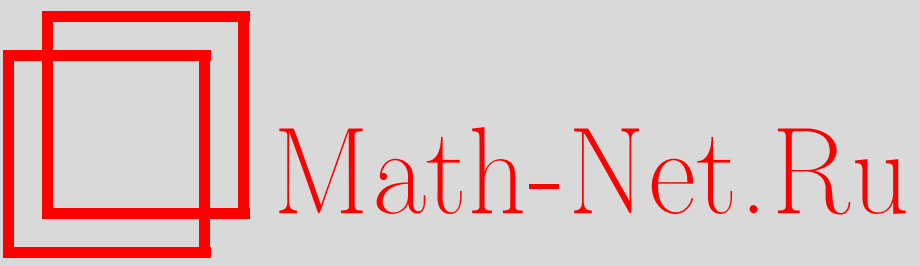

В. В. Стружанов, Об остаточных напряжениях после прокатки и расслоении двухслойных полос, Вестн. Сам. гос. техн. ун-та. Сер. Физ.-мат. науки, 2010, выпуск 5(), 55-63

DOI: https://doi.org/10.14498/vsgtu823

Использование Общероссийского математического портала Math-Net.Ru подразумевает, что вы прочитали и согласны с пользовательским соглашением

http: //www. mathnet.ru/rus/agreement

Параметры загрузки:

IP : 34.229 .108 .108

26 апреля 2023 г., 13:03:22 
УДК 539.3

\section{ОБ ОСТАТОЧНЫХ НАПРЯЖЕНИЯХ ПОСЛЕ ПРОКАТКИ И РАССЛОЕНИИ ДВУХСЛОЙНЫХ ПОЛОС}

\section{В. В. Стружанов}

Институт машиноведения УрО РАН, 620219, Екатеринбург, ул. Комсомольская, 34.

E-mails: stru@imach.uran.ru

Решена задача об определении остаточных напряжений в полосе после её прокатки в одной клети прокатного стана. Показано, что наряду с продолъными остаточными напряжениями, которые являются основной причиной возникновения дефектов геометрии полосы типа волнистости или коробоватости, в полосе существуют и поперечные остаточные напряжения, которые способствуют расслоению многослойных полос. Разработана качественная модель расслоения двухслойной полосы под действием поля растягивающих поперечных остаточных напряжений при условии равенства механических свойств материалов полос. Установлена причина скачкообразного (внезапного) расслоения, а также показано, что при отсутствии разрушения (расслоения) сцепляющей полосъ слой может быть существенно ослаблен.

Ключевые слова: однослойные и многослойные полосъ, прокатка, обжатие, первоначальные деформации, остаточные напряжсения, расслоение.

Введение. Остаточные напряжения, возникающие в полосе после её прокатки в клетях прокатного стана, оказывают существенное влияние на качество готового продукта. Например, полоса может потерять плоскостность (планшетность) из-за высокого уровня продольных сжимающих остаточных напряжений в некоторых её областях $[1,2]$. В данной работе приведено решение задачи по определению остаточных напряжений в полосе после её обжатия в одной клети. Установлено наличие не только продольных остаточных напряжений, но и поперечных, которые играют существенную роль в расслоении многослойных полос.

1. Остаточные напряжения в полосе. Рассмотрим технологическую операцию прокатки достаточно тонкой полосы в одной клети прокатного стана. С полосой свяжем систему координат (рис. 1), начало которой поместим в центр поперечного сечения полосы, ось $O x_{1}$ направим вдоль полосы в сторону направления прокатки, ось $O x_{2}$ - поперёк полосы, ось $O x_{3}$ - перпендикулярно первым двум осям таким образом, чтобы орты осей координат образовывали правую тройку векторов (декартова система координат). Поперечное сечение подката представляет собой фигуру, симметричную относительно осей $O x_{2}$ и $O x_{3}$. Аналогичной симметрией обладает и поперечное сечение после прокатки. Следовательно, система координат неизменна.

Ширина полосы не изменяется после прохождения её под валками и равна $2 b$ (уширением пренебрегаем). Свойства материала не зависят от месторасположения материальной точки в системе координат (изотропность и однородность). Геометрия подката и межвалковой щели, трение и другие меха-

Валерий Владимирович Стружанов (д.ф.-м.н., проф.), главный научный сотрудник, лаб. микромеханики материалов. 
нические и геометрические параметры процесса неизменны. Таким образом, имеет место установившийся процесс.

Мысленно рассечём подкат вдоль прямых, параллельных оси $O x_{1}$ и отстоящих друг от друга на расстоянии $d x_{2}$, плоскостями, параллельными координатной плоскости $O x_{1} x_{3}$, на параллелепипеды с основанием в виде криволинейных трапеций, непрямые стороны которой заданы симметричной относительно осей $O x_{2}$ и $O x_{3}$ функцией $h_{0}\left(x_{2}\right)$, определяющей толщину подката (рис. 1). Заменим данные трапеции прямоугольниками со сторонами $d x_{2}$ и $h_{0}^{s}$, выбирая величину $h_{0}^{s}$ таким образом, чтобы площади трапеций и прямоугольников были равны (рис. 1). В результате получаем набор длинных прямоугольных параллелепипедов. Наконец, разобьём полученные параллелепипеды по высоте на участки длиной $d x_{1}$ и будем рассматривать элементарные свободные от связей прямоугольные параллелепипеды с рёбрами $d x_{1}$, $d x_{2}, 2 h_{0}^{s}$. После прокатки, учитывая то, что уширением полосы под валками можно пренебречь, получаем прямоугольные же параллелепипеды с рёбрами $\rho d x_{1}, d x_{2}, h_{1}=2 g h_{0}^{s}$. Из условия равенства объёмов элементов до и после прокатки имеем

$$
2 d x_{1} d x_{2} h_{0}^{s}=2 \rho d x_{1} d x_{2} g h_{0}^{s} .
$$

Отсюда $\rho g=1$ и $\rho=1 / g$. Здесь $\rho, g$-соответственно коэффициенты, характеризующие степень вытяжки и обжатия. Они являются функциями от координаты $x_{2}$ и $\rho \geqslant 1,0 \leqslant g \leqslant 1$. По известным размерам свободных от связей элементов до и после прокатки определяем так называемые первоначальные деформации:

$$
\varepsilon_{11}^{*}=\rho-1=1 / g-1, \quad \varepsilon_{22}^{*}=0, \quad \varepsilon_{33}^{*}=g-1, \quad \varepsilon_{12}^{*}=\varepsilon_{13}^{*}=\varepsilon_{23}^{*}=0 .
$$

Полученные компоненты тензора деформаций не удовлетворяют условиям совместности Сен-Венана. Следовательно, в сплошной среде (полосе) эти деформации не могут быть реализованы, так как в сплошном теле могут существовать только совместные деформации. После появления первоначальных деформаций $\varepsilon_{i j}^{*}(i, j \in\{1,2,3\})$ в теле возникают препятствующие нарушению сплошности силы, составяющие самоуравновешенное поле собственных напряжений $\sigma_{i j}^{\prime \prime}$. Напряжения $\sigma_{i j}^{\prime \prime}$ инициируют появление деформаций $\varepsilon_{i j}^{\prime \prime}$ таких, что сумма деформаций

$$
\varepsilon_{i j}^{*}+\varepsilon_{i j}^{\prime \prime}=\varepsilon_{i j}^{\prime}
$$

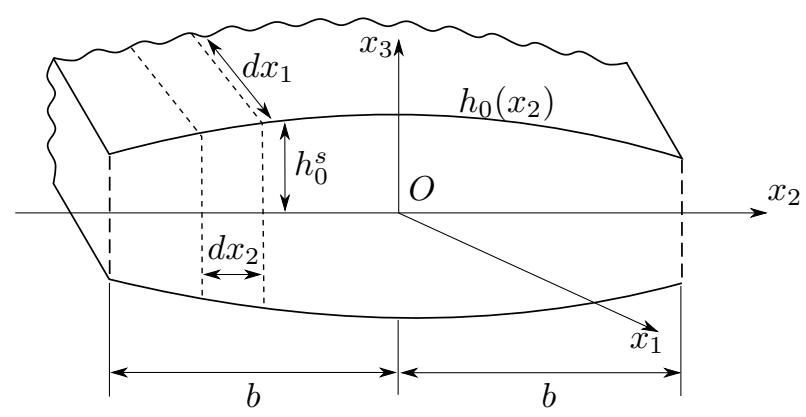

Рис. 1. Геометрия поперечного сечения подката и система координат 
уже удовлетворяет условиям совместности. Таким образом, в теле, сохранившем сплошность, будут реально присутствовать только деформации $\varepsilon_{i j}^{\prime}$ [3]. Отметим, что деформации $\varepsilon_{i j}^{\prime \prime}$ не удовлетворяют условиям совместности и связаны с напряжениями $\sigma_{i j}^{\prime \prime}$ законом Гука. Выражая из (1) деформации $\varepsilon_{i j}^{\prime}$ и подставляя их в закон Гука для данного случая, получаем

$$
\sigma_{11}^{\prime \prime}=\sigma_{11}^{\prime}-\sigma_{11}^{*}, \quad \sigma_{22}^{\prime \prime}=\sigma_{22}^{\prime}-\sigma_{22}^{*}, \quad \sigma_{33}^{\prime \prime}=\sigma_{33}^{\prime}-\sigma_{33}^{*},
$$

где

$$
\begin{array}{rll}
\sigma_{11}^{\prime}=2 \mu \varepsilon_{11}^{\prime}+\lambda \theta^{\prime}, & \sigma_{22}^{\prime}=2 \mu \varepsilon_{22}^{\prime}+\lambda \theta^{\prime}, & \sigma_{33}^{\prime}=2 \mu \varepsilon_{33}^{\prime}+\lambda \theta^{\prime}, \\
\sigma_{11}^{*}=2 \mu \varepsilon_{11}^{*}+\lambda\left(\varepsilon_{11}^{*}+\varepsilon_{33}^{*}\right), & \sigma_{22}^{*}=\lambda\left(\varepsilon_{11}^{*}+\varepsilon_{33}^{*}\right), & \sigma_{33}^{*}=2 \mu \varepsilon_{33}^{*}+\lambda\left(\varepsilon_{11}^{*}+\varepsilon_{33}^{*}\right) .
\end{array}
$$

Здесь $\lambda, \mu$ - коэффициенты Ляме, $\theta^{\prime}=\varepsilon_{11}^{\prime}+\varepsilon_{22}^{\prime}+\varepsilon_{33}^{\prime}, \sigma_{11}^{\prime}, \sigma_{11}^{*}$ - псевдонапряжения, не реализуемые в полосе.

Подставляем выражения (2) в уравнения равновесия линейной теории упругости. Имеем

$$
\frac{\partial \sigma_{11}^{\prime}}{\partial x_{1}}=0, \quad \frac{\partial \sigma_{2}^{\prime}}{\partial x_{2}}=-\lambda \frac{1-g^{2}}{g^{2}} \frac{d g}{d x_{2}}, \quad \frac{\partial \sigma_{33}^{\prime}}{\partial x_{3}}=0 .
$$

Кроме того, должны быть удовлетворены условия совместности Сен-Венана для деформаций $\varepsilon_{i j}^{\prime}$. Выражая эти деформации через напряжения $\sigma_{i j}^{\prime}$ по формулам (3) и подставляя в уравнение совместности, получаем уравнения

$$
\begin{aligned}
& \frac{1}{E} \frac{\partial^{2}}{\partial x_{2}^{2}}\left[\sigma_{11}^{\prime}-\nu\left(\sigma_{22}^{\prime}+\sigma_{33}^{\prime}\right)\right]+\frac{1}{E} \frac{\partial^{2}}{\partial x_{1}^{2}}\left[\sigma_{22}^{\prime}-\nu\left(\sigma_{11}^{\prime}+\sigma_{33}^{\prime}\right)\right]=0, \\
& \frac{1}{E} \frac{\partial^{2}}{\partial x_{3}^{2}}\left[\sigma_{11}^{\prime}-\nu\left(\sigma_{22}^{\prime}+\sigma_{33}^{\prime}\right)\right]+\frac{1}{E} \frac{\partial^{2}}{\partial x_{1}^{2}}\left[\sigma_{33}^{\prime}-\nu\left(\sigma_{11}^{\prime}+\sigma_{22}^{\prime}\right)\right]=0, \\
& \frac{1}{E} \frac{\partial^{2}}{\partial x_{3}^{2}}\left[\sigma_{22}^{\prime}-\nu\left(\sigma_{11}^{\prime}+\sigma_{33}^{\prime}\right)\right]+\frac{1}{E} \frac{\partial^{2}}{\partial x_{2}^{2}}\left[\sigma_{33}^{\prime}-\nu\left(\sigma_{11}^{\prime}+\sigma_{22}^{\prime}\right)\right]=0 .
\end{aligned}
$$

Здесь $E, \nu$ - соответственно модуль Юнга и коэффициент Пуассона.

Систему (4)-(5) будем решать, используя полуобратный метод [4]. Сделаем предположение о том, что все псевдонапряжения $\sigma_{i j}^{\prime}$ зависят только от координаты $x_{2}$. В этом случае уравнения (4) удовлетворяются, если $\sigma_{22}^{\prime}=$ $=\lambda(1-g)^{2} / g$. Из уравнений (5) остаются только два уравнения:

$$
\frac{\partial^{2}}{\partial x_{2}^{2}}\left[\sigma_{11}^{\prime}-\nu\left(\frac{\lambda(1-g)^{2}}{g}+\sigma_{33}^{\prime}\right)\right]=0, \quad \frac{\partial^{2}}{\partial x_{2}^{2}}\left[\sigma_{33}^{\prime}-\nu\left(\frac{\lambda(1-g)^{2}}{g}+\sigma_{11}^{\prime}\right)\right]=0 .
$$

Интегрируя данную систему, находим после необходимых преобразований

$$
\begin{aligned}
& \sigma_{11}^{\prime}=\frac{\lambda^{2}(1-g)^{2}}{g(\lambda+2 \mu)}+C_{1} x_{2}+C_{2}=\frac{\nu}{1-\nu} \sigma_{22}^{\prime}+C_{1} x_{2}+C_{2}, \\
& \sigma_{33}^{\prime}=\frac{\lambda^{2}(1-g)^{2}}{g(\lambda+2 \mu)}+C_{3} x_{2}+C_{4}=\frac{\nu}{1-\nu} \sigma_{22}^{\prime}+C_{3} x_{2}+C_{4} .
\end{aligned}
$$


Таким образом, напряжения $\sigma_{11}^{\prime}, \sigma_{22}^{\prime}, \sigma_{33}^{\prime}$ удовлетворяют уравнениям краевой задачи.

Подставляя найденные значения псевдонапряжений $\sigma_{i j}^{\prime}$ в равенство (2), получаем выражения для остаточных напряжений:

$$
\begin{gathered}
\sigma_{11}^{\prime \prime}=C_{1} x_{2}+C_{2}-\frac{2 \lambda \mu(1-g)^{2}}{g(\lambda+2 \mu)}-2 \mu \frac{1-g}{g}=C_{1} x_{2}+C_{2}-\frac{1-2 \nu}{1-\nu} \sigma_{22}^{\prime}-\frac{E}{1+\nu} \varepsilon_{11}^{*}, \\
\sigma_{33}^{\prime \prime}=C_{3} x_{2}+C_{4}-\frac{2 \lambda \mu(1-g)^{2}}{g(\lambda+2 \mu)}+2 \mu(1-g)=C_{3} x_{2}+C_{4}-\frac{1-2 \nu}{1-\nu} \sigma_{22}^{\prime}-\frac{E}{1+\nu} \varepsilon_{33}^{*}, \\
\sigma_{22}^{\prime \prime}=\sigma_{12}^{\prime \prime}=\sigma_{13}^{\prime \prime}=\sigma_{23}^{\prime \prime}=0 .
\end{gathered}
$$

Для определения постоянных $C_{1}, C_{2}, C_{3}, C_{4}$ воспользуемся сначала тем, что напряжение $\sigma_{11}^{\prime \prime}$ на торце полосы должно удовлетворять граничным условиям в смысле Сен-Венана, то есть главный вектор и главный момент напряжения $\sigma_{11}^{\prime \prime}$ по ширине полосы должны равняться нулю:

$$
\int_{-b}^{b} \sigma_{11}^{\prime \prime} d x_{2}=0, \quad \int_{-b}^{b} x_{2} \sigma_{11}^{\prime \prime} d x_{2}=0
$$

Отсюда

$$
\begin{gathered}
C_{1}=\frac{3}{2 b^{3}} \int_{-b}^{b} x_{2}\left[\frac{2 \lambda \mu(1-g)^{2}}{g(\lambda+2 \mu)}+2 \mu \frac{1-g}{g}\right] d x_{2}, \\
C_{2}=\frac{1}{2 b} \int_{-b}^{b}\left[\frac{2 \lambda \mu(1-g)^{2}}{g(\lambda+2 \mu)}+2 \mu \frac{1-g}{g}\right] d x_{2} .
\end{gathered}
$$

Торцы полосы свободны от напряжений $\left(\sigma_{22}^{\prime \prime}=0\right)$. Что касается напряжения $\sigma_{33}^{\prime \prime}$, то исходя из сделанных предположений оно является средним тех напряжений, которые распределены по толщине полосы. В этом случае должны выполняться следующие равенства:

$$
\int_{-b}^{b} \sigma_{33}^{\prime \prime} d x_{2}=0, \quad \int_{-b}^{b} x_{2} \sigma_{33}^{\prime \prime} d x_{2}=0
$$

Отсюда

$$
\begin{gathered}
C_{3}=\frac{3}{2 b^{3}} \int_{-b}^{b} x_{2}\left[\frac{2 \lambda \mu(1-g)^{2}}{g(\lambda+2 \mu)}-2 \mu(1-g)\right] d x_{2} \\
C_{4}=\frac{1}{2 b} \int_{-b}^{b}\left[\frac{2 \lambda \mu(1-g)^{2}}{g(\lambda+2 \mu)}-2 \mu(1-g)\right] d x_{2} .
\end{gathered}
$$

Подставляя теперь выражения для псевдонапряжений $\sigma_{i j}^{\prime}$ в закон Гука изотропного материала, находим совместные деформации, реализованные в полосе. Они определяются такими формулами:

$$
E \varepsilon_{11}^{\prime}=\left(C_{1}-\nu C_{3}\right) x_{2}+C_{2}-\nu C_{4},
$$




$$
\begin{gathered}
E \varepsilon_{22}^{\prime}=\frac{(1+\nu)(1-2 \nu)}{(1-\nu)} \sigma_{22}^{\prime}-\nu\left(C_{1} x_{2}+C_{2}+C_{3} x_{2}+C_{4}\right), \\
E \varepsilon_{33}^{\prime}=\left(C_{3}-\nu C_{1}\right) x_{2}+C_{4}-\nu C_{2} .
\end{gathered}
$$

Тогда деформации, связанные с остаточными напряжениями законом Гука, следующие:

$$
\begin{aligned}
E \varepsilon_{11}^{\prime \prime}= & \left(C_{1}-\nu C_{3}\right) x_{2}+C_{2}-\nu C_{4}-E \frac{1-g}{g}, \quad E \varepsilon_{22}^{\prime \prime}=E \varepsilon_{22}^{\prime}, \\
& E \varepsilon_{33}^{\prime \prime}=\left(C_{3}-\nu C_{1}\right) x_{2}+C_{4}-\nu C_{2}-E(q-1) .
\end{aligned}
$$

В заключение отметим, что при $g=$ const (геометрические размеры поперечных сечений полосы до и после прокатки подобны) получаем

$$
C_{1}=C_{3}=0, \quad C_{2}=\frac{1-2 \nu}{1-\nu} \sigma_{22}^{\prime}+\frac{E}{1+\nu} \varepsilon_{11}^{*}, \quad C_{3}=\frac{1-2 \nu}{1-\nu} \sigma_{22}^{\prime}+\frac{E}{1+\nu} \varepsilon_{33}^{*}
$$

и, следовательно, остаточные напряжения в полосе отсутствуют [1,2]. Деформации $\varepsilon_{i j}^{\prime \prime}=0$, а совместные деформации определяются формулами

$$
\varepsilon_{11}^{\prime}=\frac{1-g}{g}=\mathrm{const}, \quad \varepsilon_{22}^{\prime}=0, \quad \varepsilon_{33}^{\prime}=q-1=\mathrm{const} .
$$

2. Распределение остаточных напряжений в частном случае. Приведём распределения напряжений и деформаций в полосе, когда поперечное сечение подката имеет прямоугольную форму, а межвалковая щель такова, что $g=-a / b^{2} x_{2}^{2}+1$. В центре полосы обжатия нет $\left(\varepsilon_{11}^{*}=\varepsilon_{22}^{*}=\varepsilon_{33}^{*}=0\right)$. Таким образом, данная функция $g\left(x_{2}\right)$ задаёт лишь неравномерность первоначальных деформаций, которая только и является причиной возникновения остаточных напряжений (при $g=$ const напряжения не возникают).

Вычисляя постоянные $C_{1}, C_{2}, C_{3}, C_{4}$ находим, что $C_{1}=C_{3}=0$. Далее, произведя необходимые действия, получаем

$$
\begin{gathered}
C_{2}=\frac{E}{1-\nu^{2}}\left(-\frac{a \nu}{3}-1-\frac{1}{2 \sqrt{a}} \ln (|1-\sqrt{a}|)+\frac{1}{2 \sqrt{a}} \ln (|1+\sqrt{a}|)\right), \\
C_{4}=\frac{E}{1-\nu^{2}}\left(-\nu-\frac{a}{3}-\frac{\nu}{2 \sqrt{a}} \ln (|1-\sqrt{a}|)+\frac{\nu}{2 \sqrt{a}} \ln (|1+\sqrt{a}|)\right) .
\end{gathered}
$$

Если $E=19,6 \cdot 10^{4}$ МПА, $\nu=0,3, b=30$ мм, $a=0,01$, то $C_{2}=507,44$, $C_{4}=-501,76$. Эпюры остаточных напряжений показаны на рис. 2.

Отметим, что полученные в примере высокие уровни напряжений обусловлены обжатием, достаточно большим по величине и, как правило, не реализуемом на практике. При $a \rightarrow 0$ напряжения $\sigma_{11}^{\prime \prime}, \sigma_{33}^{\prime \prime} \rightarrow 0$.

3. Модель расслоения двухслойной полосы. Известно [2], что продольные сжимающие остаточные напряжения на краях полосы могут приводить к дефекту типа продольной волнистости. Другим дефектом является расслоение полосы [5], вероятность которого возрастает при прокатке многослойных полос [6], когда силы сцепления слоёв много меньше прочности материала. Рассмотрим одну модель, объясняющую расслоение двухслойной полосы в предположении о равенстве механических свойств материалов полос, когда 


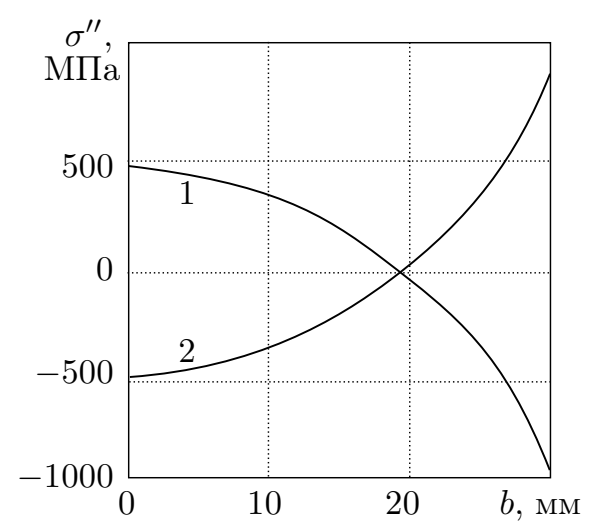

Рис. 2. Эпюры остаточных напряжений: 1 - эпюра продольных напряжений $\sigma_{11}^{\prime \prime}$; 2 - эпюра поперечных напряжений $\sigma_{33}^{\prime \prime}$

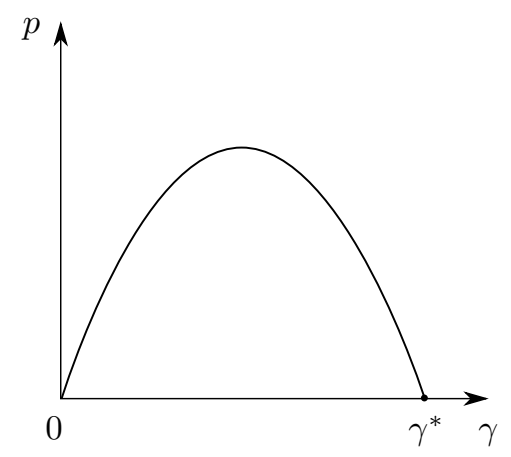

Рис. 3. Схематическая диаграмма разрыва скрепляющего слоя эпюра остаточных поперечных напряжений подобна эпюре, изображённой на рис. 2 (кривая 2).

Пусть между слоями имеется тонкий скрепляющий слой. Прочность его на отрыв определяется полной диаграммой $p(\gamma)$ (см. рис. 3), причём $p=$ $=k \gamma\left(\gamma^{*}-\gamma\right)$. Здесь $\gamma(\mathrm{Mм})-$ раздвижение берегов скрепляющего слоя, $\gamma^{*}$ - предельная величина этого раздвижения; $k\left(\mathrm{MПа} / \mathrm{Mм}^{2}\right)-$ коэффициент; $p$ (МПа) - величина растягивающей силы, приходящаяся на единицу площади. При сжатии полагаем, что в силу незначительной толщины скрепляющего слоя его берега не сдвигаются.

Для упрощения математических выкладок, преследуя цель получения только качественной картины влияния поперечных остаточных напряжений на расслоение двухслойной полосы, будем принимать во внимание только поперечные напряжения и деформации, а остальными напряжениями и деформациями пренебрегаем. Кроме того, заменим кривую 2 на рис. 2 прямой, проходящей через точку $x_{2}=b / 2$, т. е. напряжение $\sigma_{33}^{\prime \prime}=E \varepsilon_{33}^{\prime \prime}=E \alpha\left(x_{2}-b / 2\right)$.

Тогда раздвижение слоёв $\gamma=\beta\left(x_{2}-b / 2\right)$. Здесь $\alpha\left(\mathrm{мm}^{-1}\right)-$ коэффициент, характеризующий величину напряжений, $\beta$-безразмерный коэффициент, определяющий величину расхождения слоёв.

Рассмотрим часть двухслойной полосы высотой $l=1$ мм и шириной $h=$ $=1$ мм (см. рис. 4), на которую действуют заданные выше поперечные напряжения. Параметр $\alpha$ считаем параметром управления (задаваемая вели-

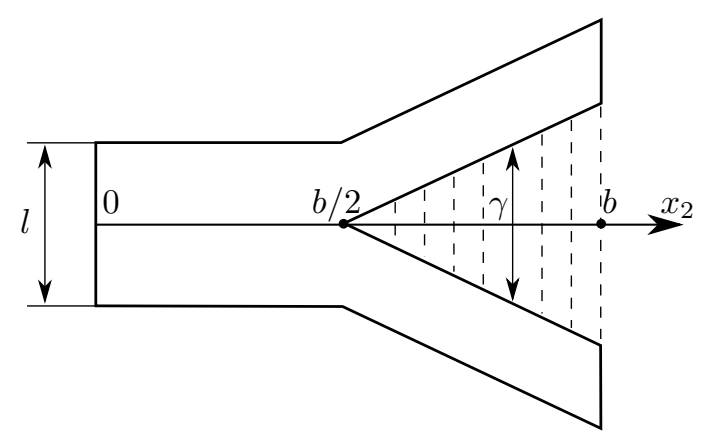

Рис. 4. Элемент двухслойной полосы под напряжением (вид спереди) 
чина), а параметр $\beta$ - параметром состояния, величина которого зависит от значения $\alpha$. В результате действия напряжений в области растяжения слои начинают расходиться. С увеличением величины раздвижения деформации $\varepsilon_{33}^{\prime \prime}$ уменьшаются и уровень напряжений падает. Их значения определяются теперь выражением $\sigma_{33}^{\prime \prime}=E\left(\alpha-\frac{\beta}{l}\right)\left(x_{2}-\frac{b}{2}\right)$. Здесь уже $\varepsilon_{33}^{\prime \prime}=\left(\alpha-\frac{\beta}{l}\right)\left(x_{2}-\frac{b}{2}\right)$.

Запишем потенциальную энергию П рассматриваемой части полосы как функцию параметров управления и состояния:

$$
\Pi=\Pi_{1}+\Pi_{2}+\Pi_{3},
$$

где $\Pi_{1}=$ const - энергия на отрезке $[0 ; b / 2]$,

$$
\begin{gathered}
\Pi_{2}=\frac{h}{2} \int_{b / 2}^{b} \sigma_{33}^{\prime \prime} \varepsilon_{33}^{\prime \prime} d x_{2}=-\frac{h}{2} E\left(\alpha-\frac{\beta}{l}\right)^{2} \int_{b / 2}^{b}\left(x_{2}-\frac{b}{2}\right)^{2} d x_{2}, \\
\Pi_{3}=h \int_{b / 2}^{b}\left(d \Pi_{3}\right) d x_{2}=h \int_{b / 2}^{b}\left(\int_{0}^{\gamma} p(\gamma) d \gamma\right) d x_{2} .
\end{gathered}
$$

После несложных преобразований получаем

$$
\Pi=\Pi_{1}+\frac{h b^{3}}{48} E\left(\alpha-\frac{\beta}{l}\right)^{2}+\frac{h k b^{3} \beta^{2}}{48}\left(\gamma^{*}-\frac{\beta b}{4}\right) .
$$

Система находится в равновесии тогда, когда [7]

$$
\frac{d \Pi}{d \beta}=-\frac{h b^{3}}{24} E\left(\alpha-\frac{\beta}{l}\right)+\frac{h k b^{3} \beta^{2}}{8}\left(\frac{\gamma^{*}}{3}-\frac{\beta b}{8}\right)=0 .
$$

Отсюда параметры $\alpha$ и $\beta$ связаны так:

$$
\alpha=\frac{\beta}{l}+\frac{k}{E} \gamma^{*} \beta-\frac{3 k}{8 E} \beta^{2} b .
$$

Процесс раздвижения приобретает скачкообразный характер, если [7]

$$
\frac{d^{2} \Pi}{d \beta^{2}}=\frac{E}{l}+k \gamma^{*}-\frac{3}{4} k \beta b=0 .
$$

Следовательно, скачок происходит после превышения параметром $\beta$ критической величины

$$
\beta^{*}=\frac{4}{3 b}\left(\frac{E}{k l}+\gamma^{*}\right)
$$

которая достигается при параметре управления, равном

$$
\alpha^{*}=\frac{4}{3 b l}\left(\frac{E}{k l}+2 \gamma^{*}+\frac{k l \gamma^{* 2}}{E}\right)-\frac{2 k}{3 E b}\left(\frac{E^{2}}{k^{2} l^{2}}+\gamma^{* 2}+2 \frac{E \gamma^{*}}{k l}\right) .
$$

Критическое значение $\alpha=\alpha^{*}$ получено после подстановки параметра $\beta=\beta^{*}$ в равенство (6). 


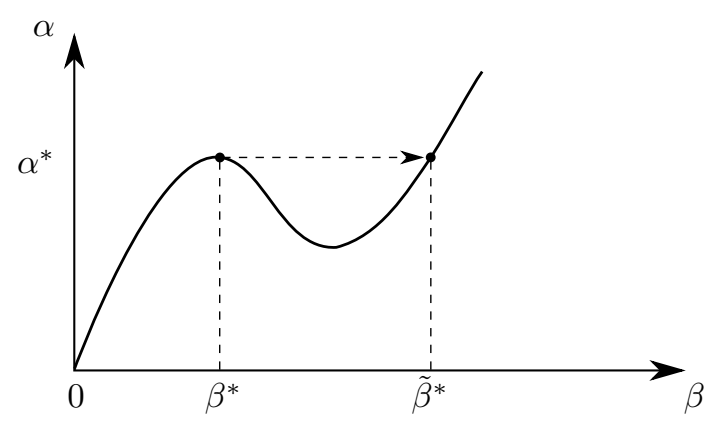

Рис. 5. Кривая равновесных состояний двухслойной полосы в зависимости от величины остаточных напряжений $\sigma_{33}^{\prime \prime}$, определяемых параметром $\alpha$

Пусть $b=30$ мм, $\gamma^{*}=10^{-4}$ мм, $k=3,92 \cdot 10^{10} \mathrm{M \Pi а} / \mathrm{мм}^{2}(\max p=98 \mathrm{M \Pi а})$, $E=1,96 \cdot 10^{5}$ МПа. Тогда из формулы (6) получаем зависимость $\alpha(\beta)$ (см. рис. 5), где $\beta^{*}=0,467 \cdot 10^{-5}, \alpha^{*}=4,9 \cdot 10^{-5}\left(\max \sigma_{33}^{\prime \prime}=565,46 \mathrm{MПа,} \max \gamma=\right.$ $\left.=0,7 \cdot 10^{-4} \mathrm{MM}\right)$.

При $\alpha=\alpha^{*}$ система находится в состоянии неустойчивого равновесия. Бесконечно малое возмущение параметра $\alpha$ приводит к тому, что реализуется переход в новое устойчивое состояние, при котором $\beta=\tilde{\beta}^{*} \approx 0,93$ $\left(\gamma_{\max }=13,95\right.$ мм $)$. Следовательно, при $\max \sigma_{33}^{\prime \prime}>565,46$ МПа происходит скачкообразное расслоение двухслойной полосы, начинающееся с её кромок.

Отметим, что при меньших напряжениях двухслойная полоса хоть и сохраняет сплошность, но связь между полосами может быть существенно ослаблена.

Работа выполнена при финансовой поддержке Программы Президиума РАН № 22 (проект № 09-П-1-1008).

\section{БИБЛИОГРАФИЧЕСКИЙ СПИСОК}

1. Рудской А.И., Лунев В.А. Теория и технология прокатного производства. - СПб.: Наука, 2008. - 527 с.

2. Двинский В.Н., Рудаков Р. В. Критическая неравномерность напряжений по ширине полосы при прокатке // Изв. вузов. Чёрная металлургия, 1981. - № 3. - С. 72-77.

3. Стружанов В. В., Миронов В. И. Деформационное разупрочнение материала в элементах конструкций. - Екатеринбург: УрО РАН, 1995. - 192 с.

4. Тимошенко С. П., Гудъер Дж. Теория упругости. - М.: Наука, 1975. - 576 с.

5. Комановский А. 3., Трощенков Н. А., Мовшович В. С. Производство легированных холоднокатанных листов. - М.: Металлургия, 1967. - 160 с.

6. Смирнова С.В., Гладковский C. В. Механические свойства и особенности разрушения слоистых металлических композитов после сварки взрывом и пакетной прокатки / В сб.: Актуальные проблемы современной науки: Труды 5-го Международного форума. Естественные науки. Ч. 1-3: Математика. Математическое моделирование. Механика. Самара: СамГТУ, 2010. - C. 214-218.

7. Gilmore R. Catastrophe Theory for Scientists and Engineers. - New York: Dover, 1993; русск. пер.: Гилмор Р. Прикладная теория катастроф: В 2-х книгах; Кн. 1. - М.: Мир, 1984. $-350 \mathrm{c}$ 


\section{MSC: 74A45}

\section{ABOUT RESIDUAL STRESSES AFTER ROLLING AND EXFOLIATION OF TWO-PLY STRIPE}

\section{V. Struzhanov}

Institute of Teoretical Engineering, Ural Branch of RAS,

34, Komsomolskaya st., Yekaterinburg, 620083, Russia.

E-mails: stru@imach.uran.ru

The residual stress definition problem was solved for stripe after rolling in one mill. Lengthwise residual stresses are basic cause of the defect initiation of stripe geometry of the buckles or corrugation type. Lengthwise residual stresses exist in the stripe with transversal residual stresses. These stresses induce the exfoliation of multi-layer stripes. The qualitative model for the two-ply stripe exfoliation under pressure of extension transversal residual stresses field was obtained subject to equality of the stripe materials stress-strain properties. The cause of the stepwise (unexpected) exfoliation was defined. The layer may be depressed essentially wanting the destruction (exfoliation) binding stripe.

Key words: single-layer and multi-layer strips, rolling, cobbing, initial deformation, residual stress, exfoliation.

Original article submitted 01/IX/2010; revision submitted $12 / \mathrm{X} / 2010$.

Valeriy V. Struzhanov (Dr. Sci. (Phys. \& Math), Chief Research Scientist, Division of Machines Mechanics \& Technology. 